\section{Xuefeng Cordyceps: insights into species diversity, life cycle and host association}

\author{
L. S. Zha ${ }^{1,3}$, T. C. Wen ${ }^{2, *}$, R. Jeewon ${ }^{4}$ Z. M. $\mathrm{Xie}^{5}$, \\ S. Boonmee ${ }^{3}$, P. D. Eungwanichayapant ${ }^{3}$ and \\ K. D. Hyde ${ }^{3}$ \\ ${ }^{1}$ School of Life Sciences, Huaibei Normal University, HB 235000 , \\ AH, China \\ ${ }^{2}$ Engineering Research Center of Southwest Bio-Pharmaceutical \\ Resources, Ministry of Education, Guizhou University, GY 550025, \\ GZ, China \\ ${ }^{3}$ Centre of Excellence in Fungal Research, Mae Fah Luang University, \\ CR 57100, Thailand \\ ${ }^{4}$ Department of Health Sciences, Faculty of Science, \\ University of Mauritius, Reduit 80837, Mauritius \\ ${ }^{5}$ Institute of Chinese Materia Medica, \\ Hunan Academy of Chinese Medicine, CS 410013, China
}

Cordyceps (= Cordyceps sensu lato) fungi are rather well known as entomopathogens and also for their medicinal importance in curing many diseases. Many cordyceps species with widespread distribution throughout China have been recorded earlier. However, their taxonomy, hosts, life cycles and the plants on which the host insects reside in are poorly understood, and have sometimes led to confusion in the proper identification of the species. In this study, Xuefeng Cordyceps collected from Xuefeng Mountain, Hunan, China are identified and an assessment of their life cycles, proper hosts and plants they are associated with is made through morphological and DNA sequence-based analyses. Morphological characterization reveals that Ophiocordyceps xuefengensis from this region as commonly accepted by mycologists and local people. Species collected also include Beauveria bassiana (sexual and asexual), Metacordyceps taii (syn. of Metarhizium guizhouense), Ophiocordyceps macroacicularis and Ophiocordyceps ramosissimum. Our result challenges previous taxonomic arrangement with regard to host identity. The hosts, previously mistaken for larvae of Endoclita nodus, are corrected herein as larvae of Endoclita davidi (Lepidoptera: Hepialidae). Ribosomal DNA sequence analyses based on the ITS regions also confirm that Clerodendrum cyrtophyllum (Lamiaceae) is the plant on which the host insects reside. Morphological illustrations, where appropriate, are given for the cordyceps species, hosts and plants. The infection mechanism and life cycle of the cordyceps are also outlined.

Keywords: Clerodendrum cyrtophyllum, cordyceps, Endoclita davidi, traditional Chinese medicine.

XUEFENG Mountain (literal meaning 'mountain with snowy top', because its top is covered with snow for most

*For correspondence. (e-mail: tingchiwen@yahoo.com) of the year) is located in west-central Hunan Province, China. It is $350 \mathrm{~km}$ long and $80-120 \mathrm{~km}$ wide with altitude varying between 240 and $1934 \mathrm{~m}$ amsl (Figure 1). The annual average temperature in this region is $12.7^{\circ} \mathrm{C}$ (varying from $-10.1^{\circ} \mathrm{C}$ to $27.3^{\circ} \mathrm{C}$ ), with relative humidity of more than $87 \%$ and abundant rainfall (annual average rainfall of $1810 \mathrm{~mm}$ ). With heavy fog and frost, winter is rather dry and cold while summer is humid and cool ${ }^{1}$.

Cordyceps (=Cordyceps sensu lato) $)^{2}$ fungi are one of the most important groups of invertebrate pathogens ${ }^{2,3}$. Currently, they include more than 900 species $^{2}$ belonging to three families (Cordycipitaceae, Ophiocordycipitaceae and partial Clavicipitaceae) in the order Hypocreales ${ }^{4}$. To date, more than 140 species are reported from China ${ }^{5}$. For a long time, Cordyceps research mainly focused on species diversity, medicinal values and biological controll $^{2-5}$, however, biology, ecology and association with hosts are rather poorly understood.

The common name 'Xuefeng Cordyceps', previously known as 'immortal herbs', had originally been recorded in the middle or late Tang Dynasty (about 1200 years ago), and the species has been intensively used to cure various cancers and other diseases, improve the physique and extend human longevity ${ }^{6}$. Although considered as a precious traditional Chinese medicine (TCM) by local Yao people, this common name was not formally reported until Ophiocordyceps xuefengensis T. C. Wen et $a l$. , the first species was introduced ${ }^{7,8}$. Molecular phylogenetic analysis indicated that $O$. xuefengensis is closely related with the important TCM Ophiocordyceps sinensis (Berk.) G. H. Sung et al. (Dong Chong Xia Cao; winter worm, summer herb) ${ }^{7}$, also their hosts are all Hepialidae larvae ${ }^{9}$. From then on, the common name Xuefeng Cordyceps came into use, in spite of the second species $O$. ramosissimum $\mathrm{T}$. C. Wen et al. being reported, which shares the same host insect that resides on the same plant as the former ${ }^{10}$.

Rather than a precious TCM, to a greater extent Xuefeng Cordyceps represent a kind of Chinese traditional culture. In this study, we analyse the species diversity of Xuefeng Cordyceps, and identify their host insects and plants on which the insects reside. We also study the biology and ecology of Xuefeng Cordyceps and their host insects. To recognize the regional cordyceps correctly, and inherit the Chinese traditional culture properly, this study provides important taxonomic, biological and ecological information.

Explorations were made in the Xuefeng Mountain region. Specimens, including the cordyceps, host insects (larvae and pupae), and plants on which the insects resided were observed in the wild and brought to the laboratory for identification. A camera (Nikon Coolpix P520) and a compound microscope (Nikon Eclipse 80i) connected with a camera (Cannon EOS 600D) were used to take photographs. Measurements were made using Tarosoft (R) Image Frame Work software. 


\section{RESEARCH COMMUNICATIONS}

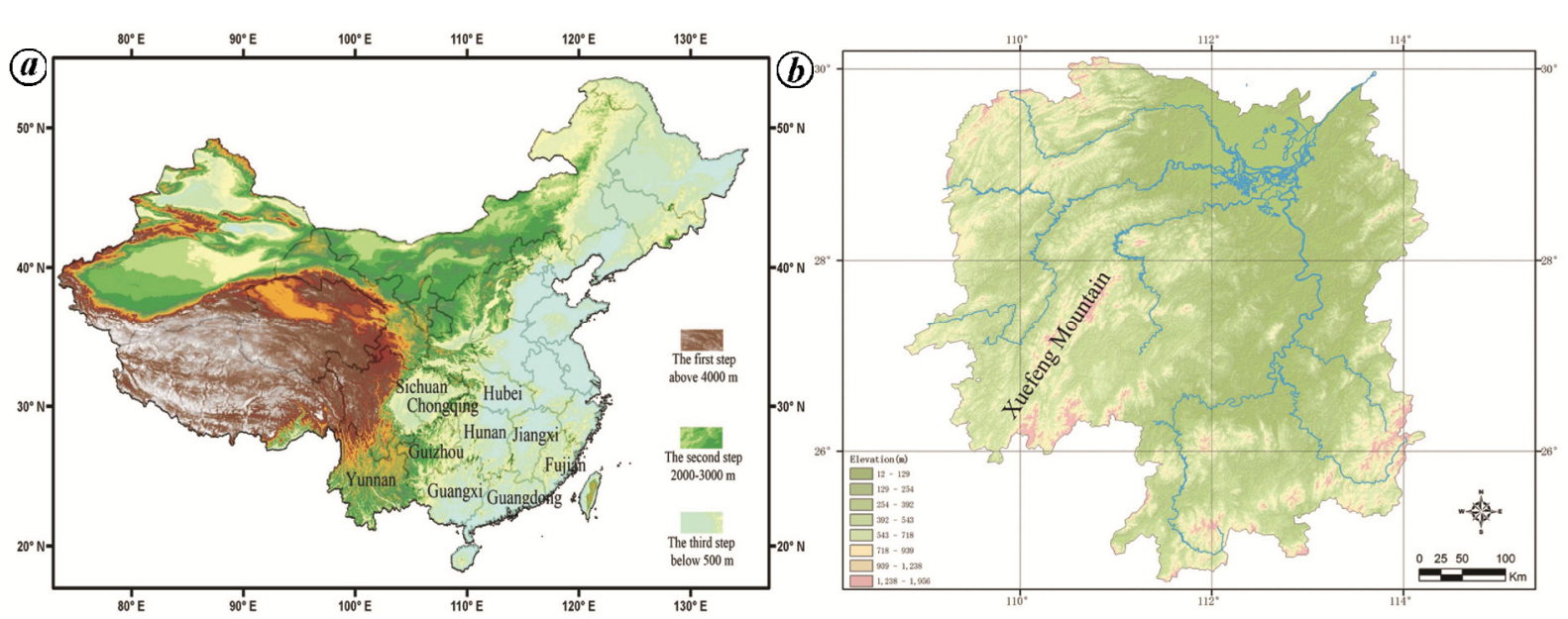

Figure 1. Topopraphic maps of China (a) and Hunan Province, China (b).

The voucher specimens are deposited in the following institutions: (i) GACP, the Herbarium of Guizhou Agricultural College, Guiyang, Guizhou, China. (ii) GZUH, the Herbarium of Guizhou University (the original Herbarium of Guizhou Agricultural College, GACP). (iii) MFLU, Centre of Excellence in Fungal Research, Mae Fah Luang University, Chiang Rai, Thailand.

Total genomic DNAs were extracted from sclerotia of the cordyceps and leaves of the plants to confirm fungus and plant identification respectively, using Fungal DNA MiniKit (E.Z.N.A. ${ }^{\text {TM }}$, Omega Biotech, CA, USA). The primer pairs ITS4/ITS5 were used for PCR amplification $^{11}$, and sequencing of ITS gene of the Cordyceps and plants. The PCR products were sequenced (GenScript Biotechnology Co., Nanjing, China). Alignments were checked visually and improved manually with care according to sequence diagrams. Clustalx1.81, ContigExpress (Invitrogen, Carlsbad, CA, USA), Chromas230 (http://www.technelysium.com.au/chromas.html) and Mega6.06 biology software were used for sequence assembly and alignment.

Blast searches were performed to reveal the closest matches of the host plants in the GenBank database that would allow the selection of appropriate taxa for phylogenetic analyses. Maximum likelihood (ML) tree was generated using RAxML ver. 8.2.8, employing a GTRGAMMA model of nucleotide substitution; other details are provided in the literature ${ }^{12,13}$. Maximum parsimony (MP) tree was constructed with PAUP*4.0b10 (ref. 14) and using the heuristic search option with TBR branch swapping and a bootstrap test of 1000 replicates; further details are outlined in the literature ${ }^{15,16}$.

In the Xuefeng Mountain region, we have so far found five Xuefeng Cordyceps species. These were dug out from basal trunks or roots of the plant $\mathrm{Cl}$. cyrtophyllum Turcz. (Lamiales: Lamiaceae), and their hosts are all larvae of Endoclita davidi Poujade (Lepidoptera: Hepialidae). The five species are recorded as follows:
(1) Beauveria bassiana (Bals.-Criv.) Vuill. ${ }^{17}$. Botrytis bassiana Bals.-Criv., Spicaria bassiana (Bals.-Criv.) Vuill., Penicillium bassianum (Bals.-Criv.) Biourge. Sexual morph, Cordyceps bassiana Z.Z. Li et al.

Material examined: Asexual morph - GACP 15101201, GACP 15101206 (Figure 2f); sexual morph-GACP 14071004, GACP 16080601 (Figure $2 e$ ).

Notes: Though the asexual B. bassiana and the sexual $C$. bassiana are conspecific ${ }^{17,18}$, herein we habitually separate them for discussions. B. bassiana is common in the wild, but it has seldom been found in trunks or roots of plants. Notably, in all entomopathogenic fungi, only $B$. bassiana and $O$. sinensis are included in the Chinese Pharmacopoeia $^{19}$. Unlike the common B. bassiana, C. bassiana is rarely collected in the wild. A third C. bassiana specimen (GACP 16041002) also grew on a Hepialidae larva, but it was dug out from soil (Guizhou, China) instead of from a tree trunk or root. The sequencing results further substantiate that $C$. bassiana is the sexual morph of B. bassiana.

(2) Metarhizium guizhouense Q.T. Chen \& H.L. $\mathrm{Guo}^{20,21}$. Metarhizium taii Z.Q. Liang \& A. Y. Liu, Metarhizium taii var. Chongqingensis Y.H. Yang et al., Metarhizium taii var. taii Z.Q. Liang \& A.Y. Liu.

Sexual morph, Cordyceps taii Z. Q. Liang \& A. Y. Liu, Metacordyceps taii (Z.Q. Liang \& A.Y. Liu) G.H. Sung et al. Material examined: GACP 1229 (Figure 2d) and GACP 1230.

Notes: M. guizhouense was originally isolated from a dead Hepialidae larva in Guizhou, China ${ }^{20}$. Liang et al. ${ }^{22}$ introduced $C$. taii and its asexual morph Metarhizium taii also on Lepidoptera larvae from Guizhou. Sung et al. ${ }^{4}$ introduced the genus Metacordyceps and revised C. taii to Metacordyceps taii. Kepler et al. ${ }^{23}$ revised Metacordyceps as a synonym of Metarhizium. Huang et al. ${ }^{24}$ treated Metarhizium taii/C. taii as synonyms of Metarhizium anisopliae (Metschn.) Sorokīn based on ITS sequence data, but these placements were rejected by Bischoff et $a .^{21}$, 

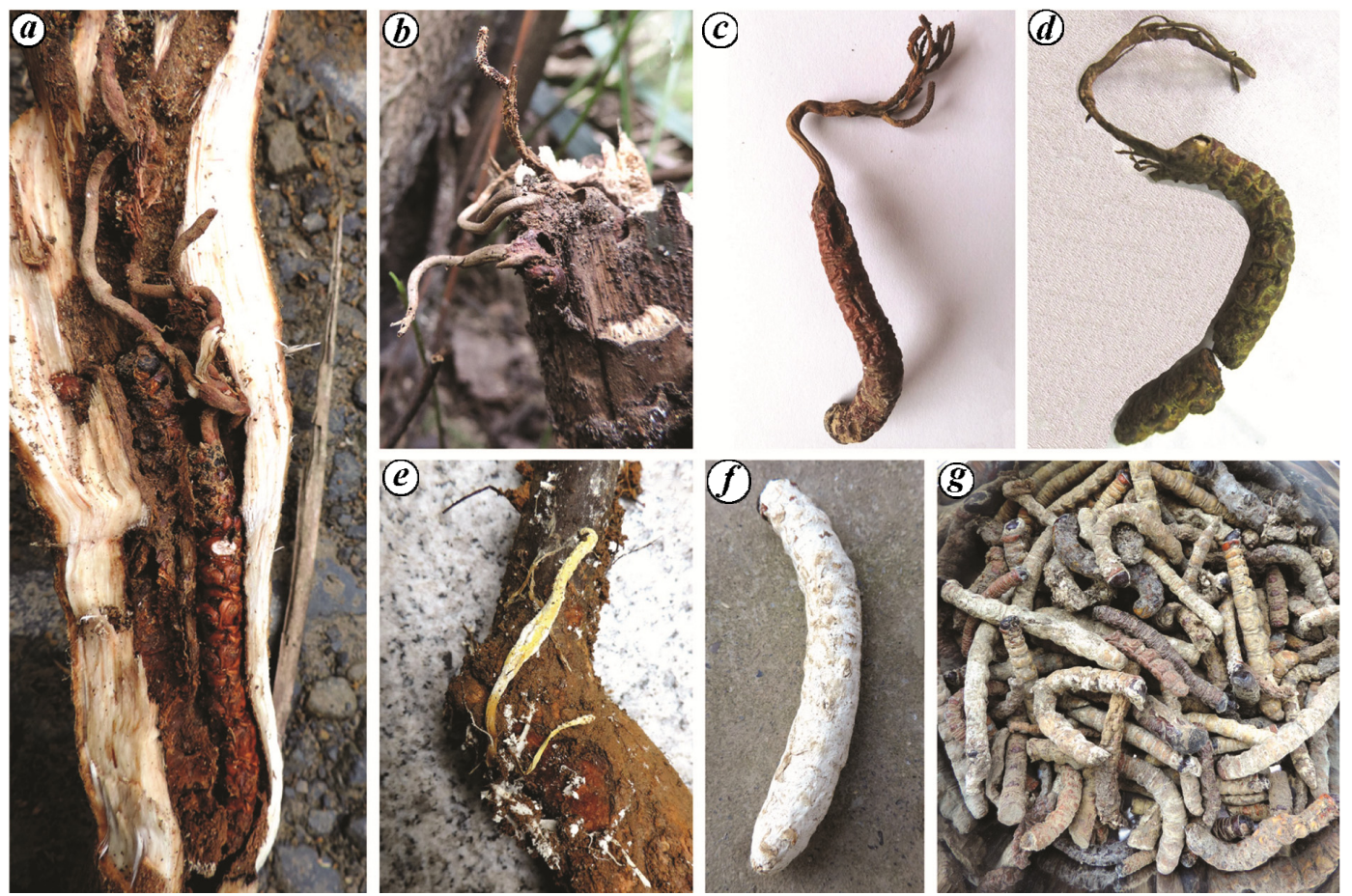

Figure 2. Species of Xuefeng Cordyceps. a, b, Ophiocordyceps xuefengensis. c, Ophiocordyceps ramosissimum. $\boldsymbol{d}$, Metacordyceps taii (syn. of Metarhizium guizhouense). $\boldsymbol{e}, \boldsymbol{f}$, Beauveria bassiana, sexual and asexual morphs. $\boldsymbol{g}$, Ossified insects.

who treated Metacordyceps taii/Metarhizium taii as synonyms of $M$. guizhouense based on a multilocus phylogeny. Evidences from hosts, geographical distributions, morphologies and molecular phylogeny are all supportive of M. guizhouense, Metacordyceps taii (C. taii) and Metarhizium taii to be conspecific.

Metacordyceps taii is widely distributed in the southwestern provinces of China and has a high yield, especially in Guizhou ${ }^{2}$. This species can be collected all year around in Guizhou, but its fruiting body stage only occurs between May and August ${ }^{2}$. Generally it grows from heads of larvae or pupae of Hepialidae in the soil. This study shows that Metacordyceps taii can also grow in tree trunks or roots.

(3) Ophiocordyceps macroacicularis S. Ban, T. Sakane \& Nakagiri, 2015 (ref. 25). Material examined: GACP 15092001, GACP 15092002, GACP 15092003, GACP 15092004. These four specimens were dug out within the same plant. Specimens from other regions: GACP SG0301-GACP SG0312 (12 specimens), March to May 2016, Guizhou, China, growing from heads of larvae of Endoclita sp. living in basal tree trunks or roots, collected by local people.

Redescription - Sexual morph (Figure 3): Stromata 1-2, seldom fasciculate, slender and long, cylindrical at base, then gradually thinning upwards, unbranched, relatively smooth, brown, $80-220 \times 0.1-2.5 \mathrm{~mm}$, growing from heads of lepidopteran larvae living in basal tree trunks or roots. Stipe - lower part (below middle) sterile, sometimes distal part also sterile due to being too slender. Perithecia-superficial, vertically placed to the stipe, ovoid and compressed, apices a little coned, yellowishbrown to brown, covering middle to sub-distal part of the stipe, but sparse or absent on distal part, 390-820 $\times 250$ $440 \mu \mathrm{m}(537.4 \times 324.7 \mu \mathrm{m}$ on average $)$. Asci - hyaline, cylindrical, eight-spored, $240-310 \mu \mathrm{m}$ long $(267.6 \mu \mathrm{m}$ on average), apical cap conspicuous and thick, 5.0-7.2 (5.6 $\mu \mathrm{m}$ on average) in diameter. Ascospores - hyaline, needle-shaped, septate indistinctly, but 10-20 septa barely visible in old-mature specimens, 200-300 $\times 2.0$ $3.0 \mu \mathrm{m} \quad(253.8 \times 2.5 \mu \mathrm{m}$ on average $)$; no partspore observed. Asexual morph: Hirsutella-like ${ }^{25,26}$.

Hosts: Growing from heads of larvae of Endoclita (Hepialidae) or Cossidae living in basal tree trunks or roots, such as E. davidi living in Cl. cyrtophyllum, or Cossida sp. living in Reynoutria japonica Houtt. (Polygonaceae) ${ }^{25}$. Distribution: China (Hunan (new record), Guizhou); Japan (Kyoto) ${ }^{25}$.

Notes: Our collections were identified as $O$. macroacicularis based on two reasons: (i) morphologically, apart from only 1-2 stromata, our collections are closely similar to O. macroacicularis from Japan ${ }^{25}$; (ii) ITS sequences of our collections (GACP 15092001, GACP 15092002 and GACP SG0301) and O. macroacicularis from Japan are highly similar (differences only one or two DNA bases).

Ban et al. ${ }^{25}$ named $O$. macroacicularis to distinguish it from O. emeiensis (A.Y. Liu \& Z.Q. Liang) G. H. Sung et al. (ref. 27) because the latter has only 1-2 stromata and 

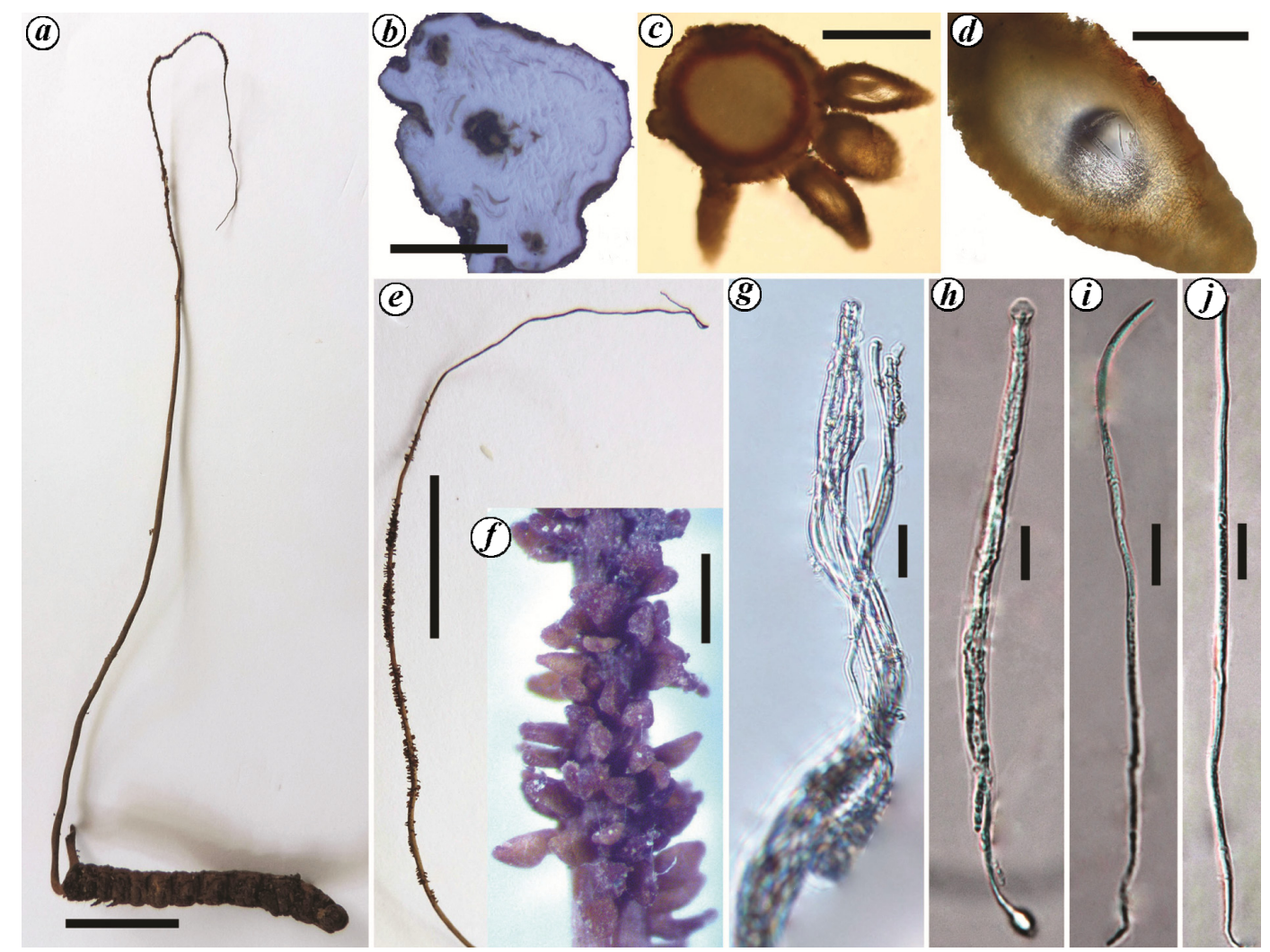

Figure 3. Ophiocordyceps macroacicularis. $\boldsymbol{a}$, Overview of stroma and the host. $\boldsymbol{b}$, Section of ossified insect. $\boldsymbol{c}$, Section of stroma. $\boldsymbol{d}$, Section of ascomata. $\boldsymbol{e}$, Distal part of stroma. $f$, Brown, superficial ascomata on stroma. $\boldsymbol{g}$, A bunch of asci twisted together. $(\boldsymbol{h})$ Mature ascus with ascospores. $\boldsymbol{i}, \boldsymbol{j}$, Hyaline and filliform ascospores with indistinct septa. Scale bar: $\boldsymbol{a}, \boldsymbol{e}=20 \mathrm{~mm}, b=2 \mathrm{~mm}, c=500 \mu \mathrm{m}, d=200 \mu \mathrm{m}, f=100 \mu \mathrm{m}, g=40 \mu \mathrm{m}, \boldsymbol{h}-\boldsymbol{j}=20 \mu \mathrm{m}$.

one distinctly different ITS sequence (AJ309347, uploaded in 2002). Notably, also having 1-2 stromata, our collections are more similar to $O$. emeiensis ${ }^{27}$. So we consider the unique ITS sequence of $O$. emeiensis (AJ309347) may be problematic, and O. macroacicularis may be a synonym of $O$. emeiensis.

(4) Ophiocordyceps ramosissimum T.C. Wen, J.C. Kang \& K.D. Hyde, 2014 (ref. 10). Material examined: MFLU 12-2165 (holotype), GZUH HN8 (Figure 2c), GZUH 2012HN2, GZUH 2012HN10 and GZUH 2012HN12 (paratypes).

Notes: Morphologically, O. ramosissimum can be easily differentiated from $O$. xuefengensis and $O$. macroacicularis by its multi-branch stromata, although the ossified insects (Figure $2 \mathrm{~g}$ ) or immature fruiting bodies are similar and not easy to distinguish.

(5) Ophiocordyceps xuefengensis T.C. Wen, R.C. Zhu, J.C. Kang \& K.D. Hyde, 2013 (ref. 7). Material examined: GZUH 2012HN14 (holotype), GZUH HN13, GZUH 2012HN11, GZUH 2012HN13, and GZUH 2012HN19 (paratypes); GACP 16090901 (Figure 2b), GACP 16092301 and GACP 16092302 (Figure $2 a$ ).

Notes: For their special medicinal value, ancient Yao people called these strange herbs growing on caterpillars as 'immortal herbs' ${ }^{6,28}$. Since the establishment of $O$. xuefengensis, the original name 'immortal herbs' has automatically been replaced by the new name 'Xuefeng Cordyceps'. In other words, Xuefeng Cordyceps represent a group of Cordyceps species, and O. xuefengensis is just one of them.

Figure 4 shows host insects of Xuefeng Cordyceps. Hosts of $O$. xuefengensis and $O$. ramosissimum were originally identified as larvae of E. nodus (Chu \& Wang) (=Phassus nodus $\mathrm{Chu} \& \mathrm{Wang})^{7,10}$, and in subsequent literature, larvae of $E$. nodus have routinely been treated as the hosts of Xuefeng Cordyceps.

Recently, in the Xuefeng Mountian region, we had dug out several hundred host specimens (including larvae, pupae and ossified insects) from tree trunks/roots. After morphological identification and comparison, the insects have been confirmed as only one Endoclita species. Fortunately, some pupae had moulted into adults successfully. The huge size, unique spots on the fore wing and a distinct protrusion in the middle of the anterior margin of the fore wing (Figure 4i), are typical characteristics which confirm that the species is E. davidi ( $=E$. giganodus $($ Chu \& Wang), $=P$. giganodus Chu \& Wang, $=E$. nankingi $(\text { Daniel), }=P \text {. nankingi Daniel; Figure } 4)^{29-31}$, instead of E. nodus that had formerly been identified and reported. 

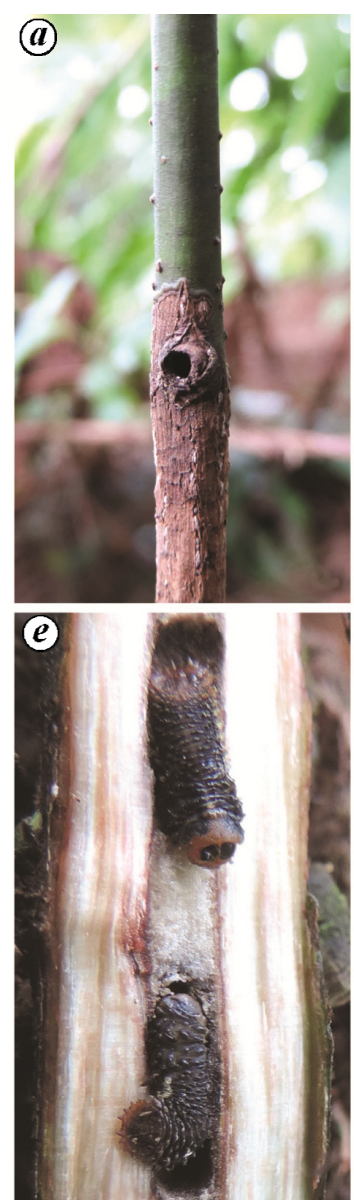

(i)

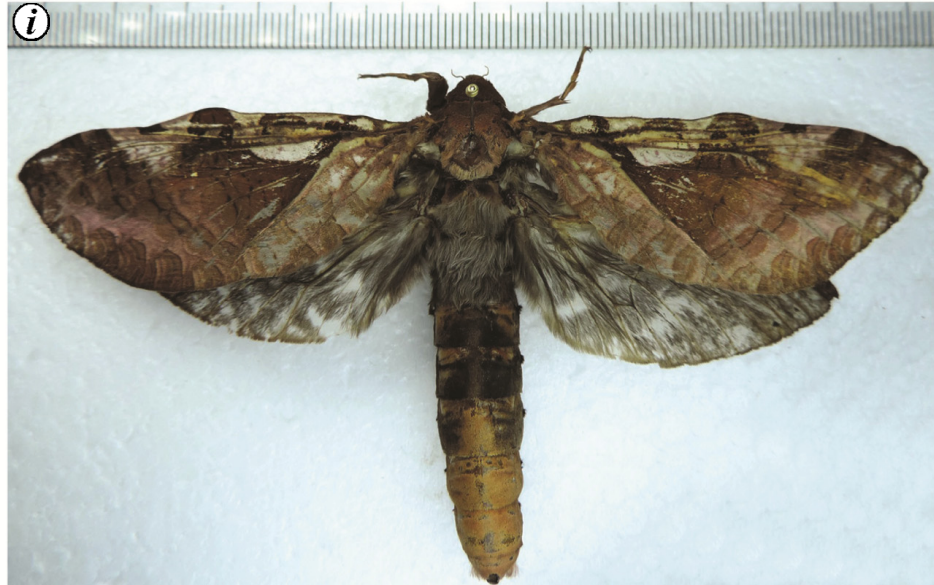

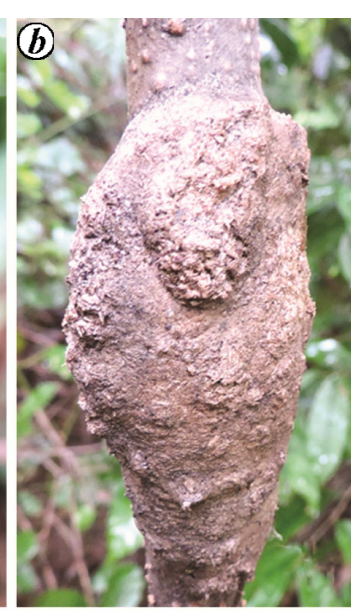

(f)

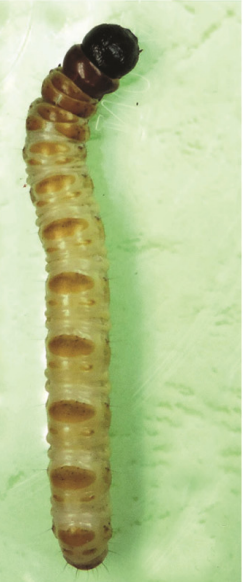

(g)

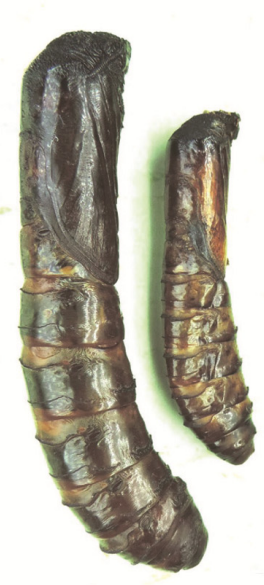

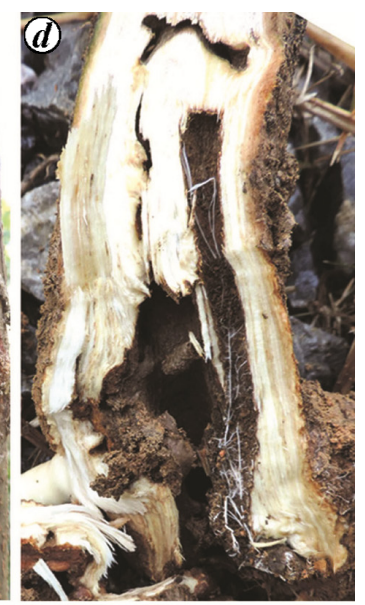
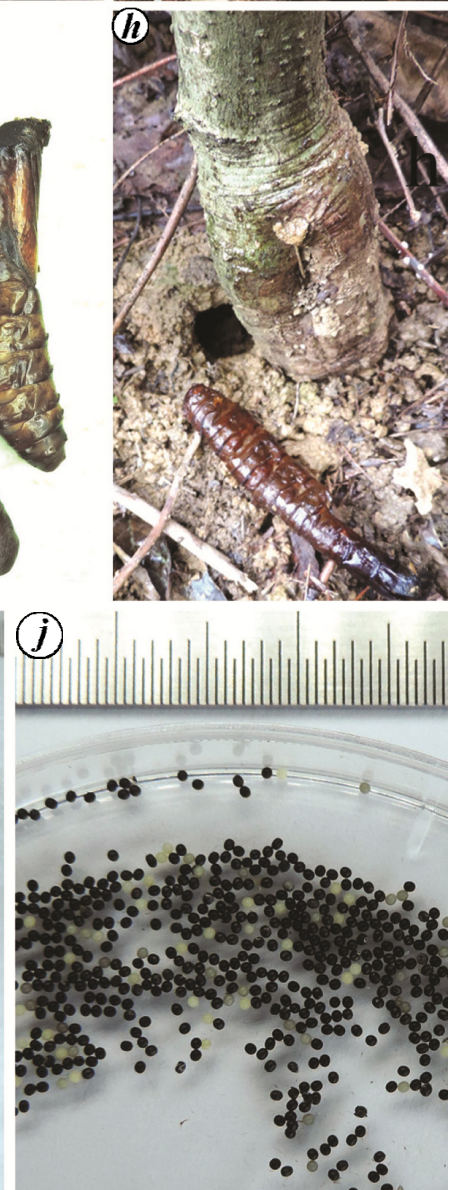

Figure 4. Endoclita davidi and its ecology. $\boldsymbol{a}$, Upper part of a tree trunk with young larvae. $\boldsymbol{b}, \boldsymbol{c}$, Frass bags produced by middle-aged larvae. $\boldsymbol{d}$, Basal trunk. $\boldsymbol{e}$, Newborn larvae. $\boldsymbol{f}$, Middle-aged larva. $\boldsymbol{g}$, Female (left) and male (right) pupae. $\boldsymbol{h}$, Pupa slough. $\boldsymbol{i}$, Female adult. $\boldsymbol{j}$, Eggs.

Biology and ecology: The newborn larvae eat young tree trunks or twigs and dig tunnels within them, and several larvae can live together in one tunnel (Figure $4 a$ and $e$ ). In the following 1-2 months they continue to eat in a downward direction and become larger. The entrance of each tunnel is covered by a large frass bag (Figure $4 b-$ $d, f)$. These large larvae are aggressive and attack/kill one another due to fierce competition; each tunnel can be occupied by only one larva. During night with no rainfall, wind and disturbance, and under relatively high temperature, the larvae crawl out and this is what makes them more susceptible to be in contact with the ascospores/ conidia of Xuefeng Cordyceps. The old-mature larvae pupate and moult in tree roots (Figure $4 d$ and $h$ ). Food shortage and disturbances can cause them to pupate and moult early, whereas lower temperature will delay their 

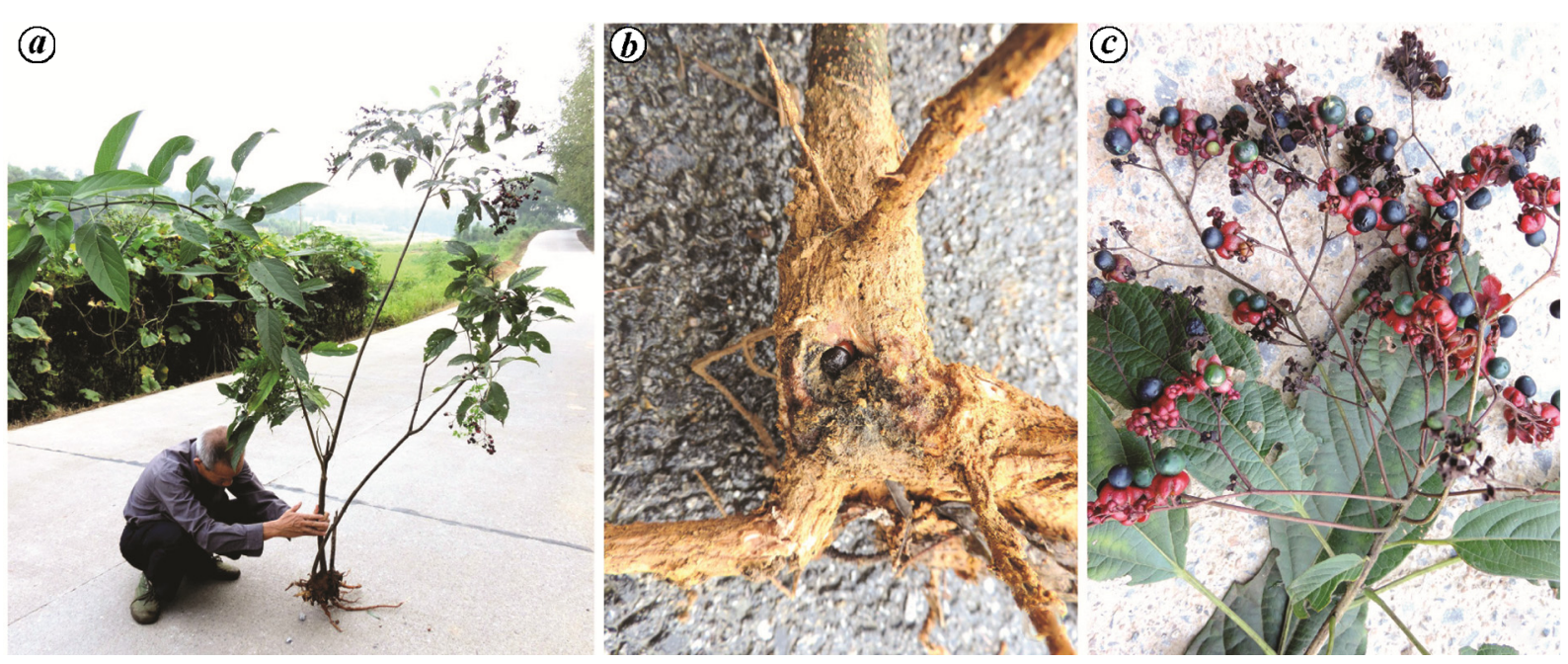

Figure 5. Clerodendrum cyrtophyllum, the host plant of Endoclita davidi in the Xuefeng Mountain region, Hunan, China. a, Plant. $\boldsymbol{b}$, Roots of the plant with E. davidi larva. $\boldsymbol{c}$, Fruits.
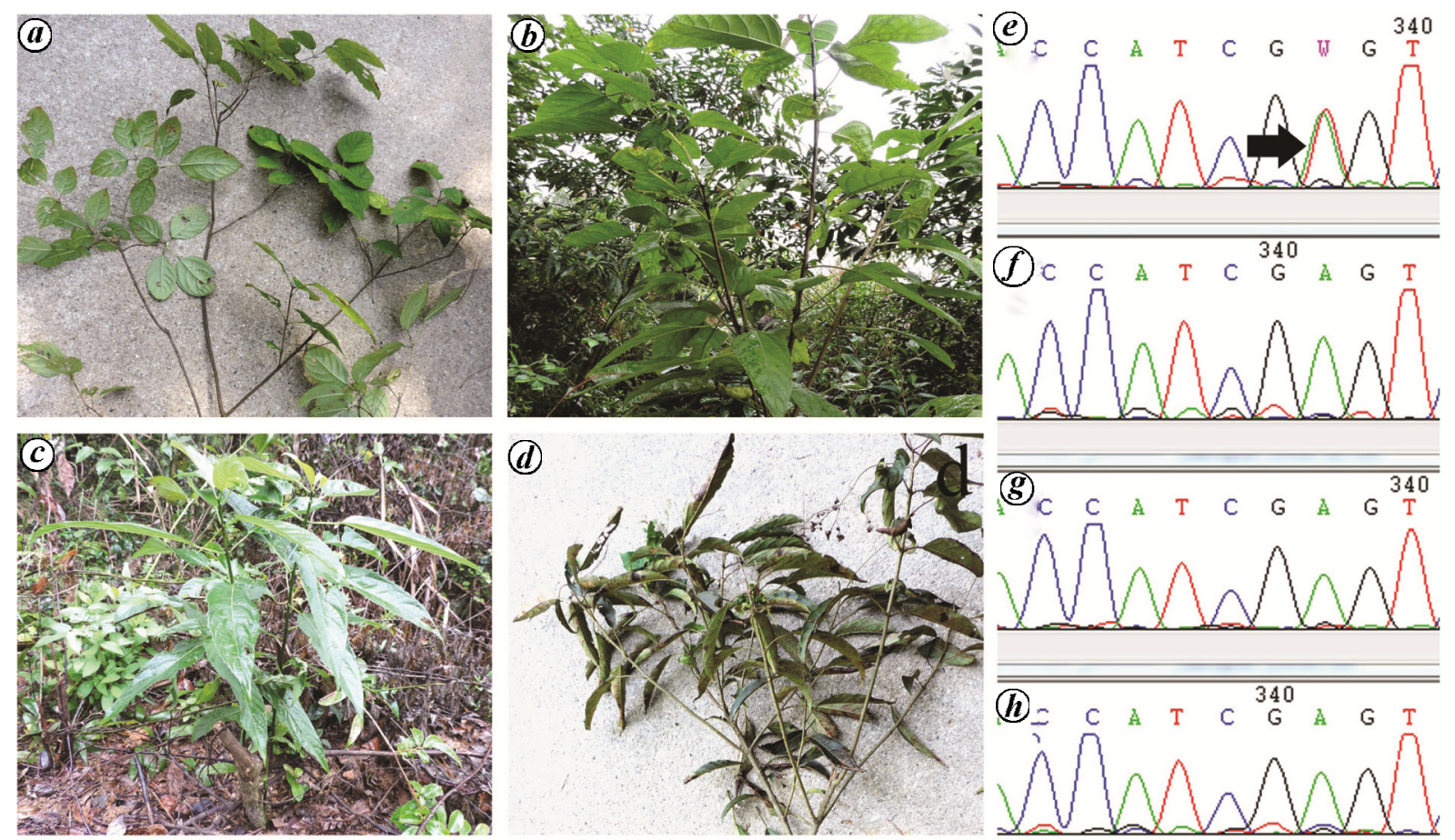

Figure 6. Morphological changes of leaves versus base variation of ITS sequence of the Clerodendrum cyrtophyllum plants. $\boldsymbol{a}$, Oval and short leaves. $\boldsymbol{b}, \boldsymbol{c}$, Long and fat leaves. $\boldsymbol{d}$, Sharp and thin leaves. $\boldsymbol{e}-\boldsymbol{h}$, Partial sequencing results of ITS sequences of the corresponding plants $(\boldsymbol{a}-\boldsymbol{d})$ with accession numbers in GenBank as KU745290, KU745291, KU745292 and KU745293 respectively.

moulting. Shortly after moulting, the insects can fly and the females begin to lay eggs (no need to mate). They lay eggs continually, especially when vibrating their wings or on being disturbed. One female can produce several thousands of eggs; the eggs are white at first, then become milky yellow and finally black (Figure $4 j$ ). The eggs fall on grasses, fallen leaves, humus layers and soil surface, but most of them will be eaten by predatory insects, mice, birds, etc. and only a few can successfully hatch. The in- sects overwinter as eggs or pupae and as a result adults can be seen two times (May and September) every year. A life cycle needs one year and the adults can live for 10 15 days. We presume that the insects are capable of parthenogenesis.

Unlike hosts of most entomopathogenic fungi, larvae of $E$. davidi live in tree trunks or roots instead of the soil, that are rarely influenced by changes in the external environment. Hence environmental factors (mainly to 


\section{RESEARCH COMMUNICATIONS}

temperature and humidity) $)^{32}$ are not the direct cause of insects being infected by Xuefeng Cordyceps. Given that the larvae are inherently aggressive, they are easily injured or even killed during fighting. This possibly renders them more vulnerable to infections and allow Cordyceps species to become opportunistic pathogens. This also explains why the infected hosts are all larvae of irregular instars instead of pupae or adults (Figure $2 \mathrm{~g}$ ), and the reason why several Cordyceps simultaneously grew in neighbouring tunnels (Figure $2 a$ ). Apart from fighting, other injuries can also cause the larvae being infected (Figure $2 b$ ).

Xuefeng Cordyceps grow slowly due to limited humidity in the plants, but they can continuously grow because both humidity and temperature are steady. Fruiting bodies of three Ophiocordyceps species can grow for 1-3 years in their plants, which is much longer than that of $B$. bassiana (sexual morph) and Metacordyceps taii. The fruiting body grows along the tunnel, until it reaches the entrance of the tunnel where it will begin to sporulate. As the largest species in Hepialidae ${ }^{31}$, the huge larva of $E$. davidi can provide sufficient nutrition for the fungus to grow, and this possibly explains why Xuefeng Cordyceps are huge. Length of the fruiting body is dependent on the length of the tunnel, and as a result some fruiting bodies can even grow up to more than $40 \mathrm{~cm}$.

Figure 5 shows host plants of the host insects. Proper identification of the host plants of $E$. davidi is always controversial. In the Xuefeng Mountain region, Huang ${ }^{6,28}$ observed three plant species but did not identify them

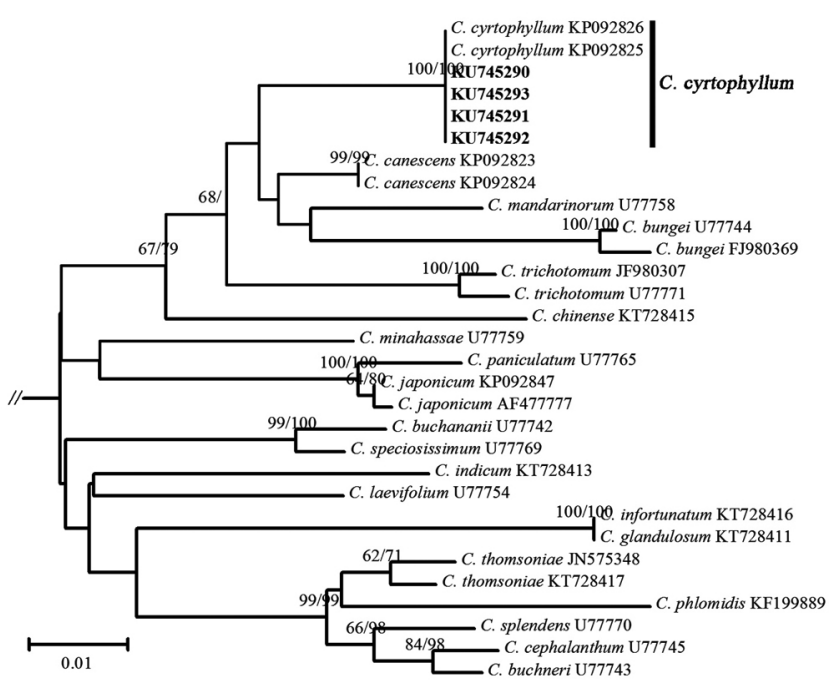

Figure 7. Maximum likelihood (ML) tree of Clerodendrum cyrtophyllum and its allies inferred from ITS rDNA gene sequences. The ITS gene dataset comprised 654 characters (including gaps), of which 187 were variable and 123 were parsimony-informative. Bootstrap support values greater than $60 \%$ for ML and maximum parsimony (MP) are indicated above the nodes and separated by "/ (ML/MP). KU745290, KU745291, KU745292 and KU745293 refer to the host plants with different leaf shapes. properly. Wen et al. ${ }^{7,10}$ reported $C l$. cyrtophyllum as the host plant, but did not give any further taxonomic and botanical information to properly validate host identity. Given the relative importance of Cordyceps in TCM or biocontrol, it is absolutely necessary to properly name the hosts and fungi concerned. In this study, a morphological comparison supplemented by DNA sequence-based data is implemented to better support any taxonomic arrangement proposed.

Depending on tunnels and frass bags produced by larvae of E. davidi, the host plants were confirmed and collected for identification. Morphologically, the plants are similar to $\mathrm{Cl}$. cyrtophyllum, but leaf shapes of the plants vary distinctly and can be classified into three types: (i) oval and short (Figure 6a); (ii) long and fat (Figure $6 b$ and $c$ ) and (iii) sharp and thin (Figure $6 d$ ). Molecular phylogenetic analysis was used to identify these plants ${ }^{33}$.

DNA sequence analyses reveal that the host plants with different leaf shapes have exactly the same ITS sequence (only one DNA base difference, see arrow in Figure $6 e-$ $h$ ), and phylogeny generated further confirms that the host plants are all $\mathrm{Cl}$. cyrtophyllum (Figure 7). It is to be noted that in the Xuefeng Mountain region, other plants such as Rhus chinensis Mill., Loropetalum chinense Oliv., Argyreia pierreana Bois and Paulownia sp. can also harbour larvae of $E$. davidi which can also crawl up to eat, but a short time later they will retreat. In trunks or roots of these plants, larvae of $E$. davidi have been rarely, and Xuefeng Cordyceps have never been discovered.

According to the present analyses, we can summarize that Xuefeng Cordyceps refer to Cordyceps fungi that grow on larvae of $E$. davidi living in basal trunks or roots of $\mathrm{Cl}$. cyrtophyllum in the Xuefeng Mountain region, Hunan, China. These Cordyceps fungi are associated with B. bassiana (asexual and sexual morphs), Metacordyceps taii (syn. of Metarhizium guizhouense), O. macroacicularis, O. ramosissimum, O. xuefengensis, etc. rather than only one species, viz. O. xuefengensis as commonly accepted by mycologists and local people.

With similar chemical composition ${ }^{34-36}$ and antitumour effect ${ }^{37}$ to the important TCM O. sinensis, Xuefeng Cordyceps have increasingly been accepted by researchers around the world. Their market price, even the ossified insects alone (Figure $2 \mathrm{~g}$ ), has already risen to US\$ $30,000 / \mathrm{kg}$ (dry weight) that is exactly the price of $O$. sinensis $^{2}$. What's more, they are huge cordyceps, generally a dry specimen has 3-5 g, while $O$. sinensis has only $0.2-0.6 \mathrm{~g}$ each. On one occasion, $120 \mathrm{~g}$ Xuefeng Cordyceps had at one time been dug out from a big tree ${ }^{6}$. Driven by the huge economic benefits, $\mathrm{Cl}$. cyrtophyllum plants are being damaged excessively and yield of Xuefeng Cordyceps was drastically decreased in recent years. However, prevailing in low altitude unlike $O$. sinensis, artificial culture of Xuefeng Cordyceps will probably be realized in the near future. 
Conflict of interest: The authors declare that they have no conflict of interest.

1. Yuan, L.-P., The Xuefeng Mountain, a famous Chinese mountain. Forest Humankind, 2014, 8, 42-49 (in Chinese).

2. Zha, L.-S. et al., An evaluation of common Cordyceps (Ascomycetes) species found in Chinese markets. Int. J. Med. Mushrooms, 2018, 20, 1149-1162.

3. Hywel-Jones, N. L., The biological diversity of invertebrate pathogenic fungi. In Biodiversity of Tropical Microfungi (ed. Hyde, K. D.), Hong Kong University Press, Hong Kong, 2001, pp. $107-120$.

4. Sung, G. H. et al., Phylogenetic classification of Cordyceps and the clavicipitaceous fungi. Stud. Mycol., 2007, 57, 5-59.

5. Wen, T.-C. et al., Metacordyceps shibinensis sp. nov. from larvae of Lepidoptera in Guizhou Province, southwest China. Phytotaxa, 2015, 226, 51-62.

6. Huang, M.-H., A Book for Exploring Xuefeng Cordyceps (Xuefeng Chongcao Tansuo Duben), Guangming Daily Publishing House, Beijing, China, 2012 (in Chinese).

7. Wen, T.-C. et al., Ophiocordyceps xuefengensis sp. nov. from larvae of Phassus nodus (Hepialidae) in Hunan Province, southern China. Phytotaxa, 2013, 123, 41-50.

8. Liu, H. et al., Studies on macroscopic and microscopic characteristics of Ophiocordyceps xuefengensis. Chin. J. Chin. Mater. Med., 2015, 40, 2820-2824 (in Chinese with English summary).

9. Wang, X.-L. and Yao, Y.-J., Host insect species of Ophiocordyceps sinensis: a review. ZooKeys, 2011, 127, 43-59.

10. Wen, T.-C. et al., Systematic analyses of Ophiocordyceps ramosissimum sp. nov., a new species from a larvae of Hepialidae in China. Phytotaxa, 2014, 161, 227-234.

11. White, T. J. et al., Amplification and direct sequencing of fungal ribosomal RNA genes for phylogenetics. In PCR Protocols: A Guide to Methods and Applications (eds Innis, M. A. et al.), Academic Press, New York, USA, 1990, pp. 315-322.

12. Jeewon, R. et al., Phylogenetic significance of morphological characters in the taxonomy of Pestalotiopsis species. Mol. Phylogenet. Evol., 2003, 27, 372-383.

13. Hongsanan, S. et al., An updated phylogeny of Sordariomycetes based on phylogenetic and molecular clock evidence. Fungal Divers., 2017, 84, 25-41.

14. Swofford, D. L., PAUP*: Phylogenetic Analysis Using Parsimony (*and Other Methods), Version 4.0b10, Sinauer Associates, Sunderland, Massachusetts, USA, 2002.

15. Cai, L., Jeewon, R. and Hyde, K. D., Molecular systematics of Zopfiella and allied genera: evidence from multigene sequence analyses. Mycol. Res., 2006, 110, 359-368.

16. Tang, A. M. C., Jeewon, R. and Hyde, K. D., Phylogenetic utility of protein (RPB2, $\beta$-tubulin) and ribosomal (LSU, SSU) gene sequences in the systematics of Sordariomycetes (Ascomycota, Fungi). Antonie van Leeuwenhoek, 2007, 91, 327-349.

17. Kepler, R. M. et al., A phylogenetically-based nomenclature for Cordycipitaceae (Hypocreales). IMA Fungus, 2017, 8, 335-353.

18. Li, Z.-Z. et al., Discovery and demonstration of the teleomorph of Beauveria bassiana (Bals.) Vuill., an important entomogenous fungus. Chin. Sci. Bull., 2001, 46, 751-753.

19. Chinese Pharmacopoeia Commission, Chinese Pharmacopoeia (Edition 10th), Vol. 1, China Medical Science Press, Beijing, China, 2015 (in Chinese).

20. Guo, H.-L. et al., Three new species of Metarhizium. Acta Mycol. Sin., 1986, 5, 177-184 (in Chinese with English summary).

21. Bischoff, J. F., Rehner, S. A. and Humber, R. A., A multilocus phylogeny of the Metarhizium anisopliae lineage. Mycologia, $2009, \mathbf{1 0 1}, 512-530$.
22. Liang, Z.-Q., Liu, A.-Y. and Liu, J.-L., A new species of the genus Cordyceps and its Metarhizium anamorph. Acta Mycol. Sin., 1991, 10, 257-262 (in Chinese with English summary).

23. Kepler, R. M. et al., Clarification of generic and species boundaries for Metarhizium and related fungi through multigene phylogenetics. Mycologia, 2014, 106, 811-829.

24. Huang, B. et al., Molecular evidence for the taxonomic status of Metarhizium taii and its teleomorph, Cordyceps taii (Hypocreales, Clavicipitaceae). Mycotaxon, 2005, 94, 137-147.

25. Ban, S. et al., Three new species of Ophiocordyceps and overview of anamorph types in the genus and the family Ophiocordyceptaceae. Mycol. Prog., 2015, 14, e1017.

26. Zhou, Y.-M. et al., A new record of the genus Ophiocordyceps from China. Microbiol. China, 2015, 42, 1710-1716 (in Chinese with English summary).

27. Liu, A.-Y., Liang, Z.-Q. and Liu, Z.-Y., Cordyceps spp. and some other entomopathogenic fungi from the Emei Mountain Preserve in China. Mycosystema, 1997, 16, 139-143 (in Chinese with English summary).

28. Huang, M.-H., An Introduction to Ecological Sociology of Xuefeng Cordyceps (Xuefeng Chongcao Shengtai Shehuixue Daolun), Guangming Daily Publishing House, Beijing, China, 2013 (in Chinese).

29. Chu, H.-F. and Wang, L.-Y., On the stem-borers of Chinese hepialids (Lepidoptera: Hepialidae). Acta Entomol. Sin., 1985, 28, 293-301 (in Chinese with English summary).

30. Nielsen, E. S. et al., Ghost-moths of the world: a global inventory and bibliography of the Exoporia (Mnesarchaeoidea and Hepialoidea) (Lepidoptera). J. Nat. Hist., 2000, 34, 823-878.

31. Zhu, H.-F., Wang, L.-Y. and Han, H.-X., Fauna Sinica (Insecta, Vol. 38, Lepidoptera, Hepialidae, Epiplemidae), Science Press, Beijing, China, 2004 (in Chinese with English summary).

32. Wen, T.-C. et al., Some entomological issues in studying entomogenous fungi. Mycosystema, 2016, 35, 1303-1309 (in Chinese with English summary).

33. Melapu, V. K. et al., A comparative phylogenetic evaluation of chloroplast ITS sequences to analyze the bioactivity in medicinal plants: a case study of Clerodendrum plant genus (Lamiaceae). Austin J. Comput. Biol. Bioinformat., 2015, 2, e1011.

34. Zhang, S.-H. et al., Identification of chemical constituents in Ophiocordyceps xuefengensis sp. nov. by HPLC-Q-TOF-MS/MS. Chin. Tradit. Herbal. Drugs, 2015, 46, 817-821 (in Chinese with English summary).

35. Zhu, R.-C. et al., Study on dynamic changes of adenosine in Ophiocordyceps xuefengensis from different harvest time. Mod. Chin. Med., 2015, 17, 1180-1183 (in Chinese with English summary).

36. Qin, Y. et al., HPLC fingerprint of Ophiocordyceps xuefengensis. J. Hunan Univ. Chin. Med., 2016, 32, 47-50 (in Chinese with English summary).

37. Zheng, B. et al., Effects of Ophiocordyceps xuefengensis on proliferation of DC-CIK cells and activity of killing HepG-2 cells by DC-CIK cells. Chin. J. Immunol., 2015, 31, 189-192 (in Chinese with English summary).

ACKNOWLEDGEMENTS. This work was supported by the National Natural Science Foundation of China (No. 31760014), and the Science and Technology Foundations of Guizhou Province, China (No. [2019]2451-3 and No. [2016]2863).

Received 13 May 2016; revised accepted 8 July 2019

doi: $10.18520 /$ cs/v117/i5/839-846 